\title{
Pneumonie à Legionella pneumophila et rhabdomyolyse
}

\section{Legionella pneumophila Pneumonia and Rhabdomyolisis}

\section{E. Hansconrad · P. Plaisance}

Reçu le 24 septembre 2018; accepté le 20 décembre 2018

(C) SFMU et Lavoisier SAS 2019

\section{Introduction}

La légionellose est une pathologie rare, environ 1600 cas notifiés en France en 2017, mais potentiellement grave avec un taux de mortalité aux alentours de $10 \%$ dans les formes sévères [1]. Nous rapportons le cas d'un patient de 65 ans ayant présenté une rhabdomyolyse compliquée d'une insuffisance rénale aiguë au décours d'une pneumonie à Legionella pneumophila.

\section{Cas clinique}

Un homme de 65 ans, en provenance d'Australie et voyageant en Europe depuis 15 jours, consulte aux urgences pour une dyspnée apparue il y a six jours et d'aggravation progressive. La dyspnée n'est pas accompagnée de toux ni de crachats ni de douleur thoracique. Une sensation fébrile est décrite par le patient. Ses antécédents sont une cardiopathie ischémique traitée par angioplastie coronarienne avec pose de stent et une hypertension artérielle. Il ne fume pas. Son traitement habituel est aspirine, lercanidipine, ramipril, métoprolol, fénofibrate.

À l'arrivée du patient aux urgences, les paramètres vitaux sont une pression artérielle à $155 / 95 \mathrm{mmHg}$, une tachycardie à $114 / \mathrm{min}$, une fièvre à $39,7^{\circ} \mathrm{C}$. La fréquence respiratoire est à $40 / \mathrm{min}$, et la saturation est à $88 \%$ en air ambiant remontant à $96 \%$ sous 31 d'oxygène. Sur le plan clinique, le patient ne présente pas de signes de choc, il est polypnéique avec un tirage sus-claviculaire, l'auscultation pulmonaire est claire et

E. Hansconrad $(\bowtie) \cdot$ P. Plaisance

Service d'accueil des urgences, hôpital Lariboisière,

Assistance publique-Hôpitaux de Paris (AP-HP),

2, rue Ambroise-Paré, F-75010 Paris, France

e-mail : erwin.hansconrad@aphp.fr

\section{P. Plaisance}

Faculté de médecine Lariboisière-Saint-Louis, université Paris-Diderot, Paris-VII,

10, avenue de Verdun, F-75010 Paris, France symétrique sans bruits surajoutés. L'examen cardiaque est normal, l'abdomen est indolore. L'électrocardiogramme retrouve une tachycardie sinusale isolée. Un bilan biologique est réalisé avec pour résultats : syndrome inflammatoire biologique CRP $183 \mathrm{mg} / \mathrm{l}$ avec hyperleucocytose $20000 / \mathrm{mm}^{3}$ avec prédominance de neutrophiles à $16200 / \mathrm{mm}^{3}$, thrombopénie à $128000 / \mathrm{mm}^{3}$, hémoglobine $15,1 \mathrm{~g} / \mathrm{dl}$, insuffisance rénale aiguë créatinine $171 \mu \mathrm{mol} / \mathrm{l}$ urée $13 \mathrm{mmol} / \mathrm{l}$, rhabdomyolyse avec CPK 2035 UI/l, cytolyse hépatique prédominant sur les ASAT à 117 UI/l, ALAT 55 UI/l, absence de cholestase, hyponatrémie à $123 \mathrm{mmol} / \mathrm{l}$, hypokaliémie à $3,0 \mathrm{mmol} / \mathrm{l}$, chlorémie à $87 \mathrm{mmol} / \mathrm{l}$, hyperlactatémie à $2,7 \mathrm{mmol} / \mathrm{l}$, bicarbonates $21 \mathrm{mmol} / \mathrm{l}$. La radiographie de thorax faite au lit (Fig. 1) retrouve un foyer de condensation alvéolaire du lobe moyen et supérieur droit. Une antigénurie légionelle et pneumocoque est réalisée aux urgences ainsi qu'un examen cytobactériologique des crachats (ECBC). Une antibiothérapie par lévofloxacine $500 \mathrm{mg} / 12$ heures et spiramycine $1,5 \mathrm{mUI} / 8$ heures est débutée aux urgences. Devant cette pneumonie sévère du lobe moyen et supérieur droit avec hypoxémie importante, signes de détresse respiratoire initiaux, hyperlactatémie et les signes extrarespiratoires à type de rhabdomyolyse, insuffisance rénale aiguë et hyponatrémie, le patient est transféré en soins continus pour la suite de la prise en charge. L'antigénurie légionelle revient positive à Legionella pneumophila de type 1, et l'ECBC revient également positif à Branhamella catarrhalis multisensible. L'évolution du patient a été favorable avec la poursuite de la biantibiothérapie introduite initialement et poursuivie pendant 14 jours. Une déclaration a été faite à l'Agence Régionale de Santé (ARS) sur la présence d'un cas de légionellose.

\section{Discussion}

La légionellose est une maladie infectieuse bactérienne, causée par un bacille à Gram négatif, dont il existe plusieurs espèces et sérogroupes. Legionella pneumophila est la plus souvent mise en cause et tout particulièrement le sérogroupe 1. La légionellose est une maladie à déclaration 


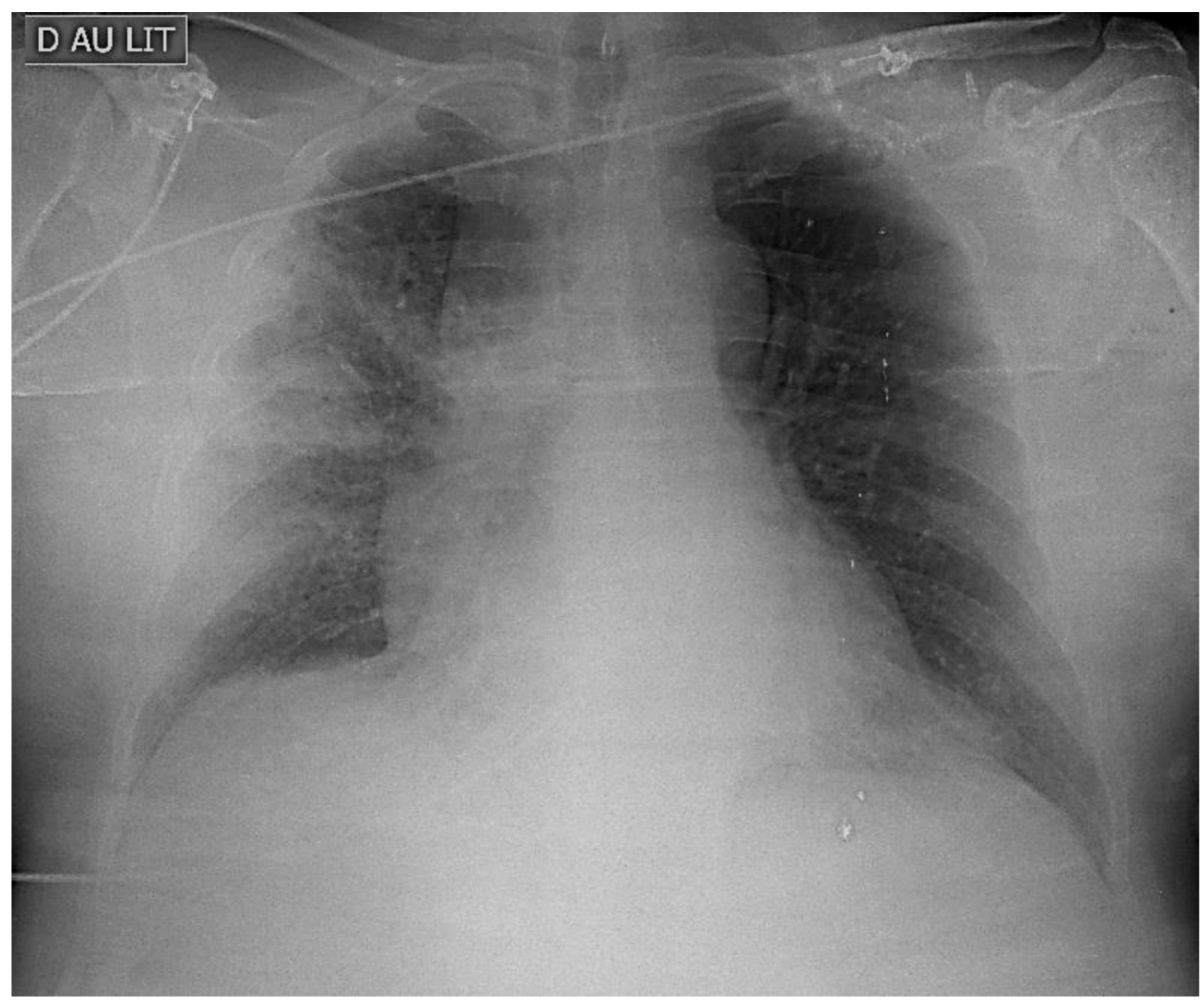

Fig. 1 Radiographie de thorax de face montrant une opacité alvéolaire du lobe moyen et supérieur droit

obligatoire en France. La contamination se fait par l'inhalation de gouttelettes d'eau infectée par le germe présent dans les systèmes de canalisation d'eau ou de climatisation [2-3]. La légionellose se manifeste par deux tableaux : la forme sporadique ou fièvre de Pontiac qui ressemble à un syndrome grippal et la forme plus sévère qui est une pneumonie pouvant conduire à une défaillance multiviscérale et au décès du patient [2-5]. La rhabdomyolyse est une complication rare de la légionellose dont le mécanisme reste inconnu [6]. La présentation sévère est signalée dans environ $15 \%$ des cas ; ses complications induisant un taux de mortalité de plus de $50 \%$ peuvent s'élever à $80 \%$ en fonction du terrain de l'hôte $[7,8]$. La légionellose n'ayant pas de spécificité radioclinique, son diagnostic doit être évoqué devant toute pneumonie. La présence d'éléments tels qu'un contexte nosocomial ou épidémique (voyage, thermes, exposition à de l'eau en aérosols), un tableau clinique évocateur (pneumonie d'allure sévère, atteinte neurologique et/ou multisystémique), la présence de signes extrapulmonaires (cytolyse hépatique, syndrome glomérulaire et/ou insuffisance rénale, rhabdomyolyse), un terrain favorisant (cancer, immunodépression) ou l'échec d'un traitement initial par bêtalactamine à visée antipneumococcique correctement prescrit sont d'autant plus en faveur d'une légionellose [9]. Le diagnostic est confirmé par la mise en culture d'un prélèvement respiratoire qui reste la méthode de référence permettant d'identifier exactement le type de Legionella pneumophila. Son inconvénient est le délai pour le résultat qui peut prendre trois à cinq jours. La recherche d'antigènes solubles urinaires (antigénurie) est une technique largement plus utilisée dans les services d'urgences. Ce test est rapide (résultat en moins d'une heure) et facilement réalisable. Il est également sensible ( $80 \%$ sur des urines concentrées) et très spécifique (99\%) [10]. Il est positif dès le début de la maladie (deux à trois jours après l'apparition des signes cliniques), et le reste pendant l'évolution (deux mois en moyenne), même après institution d'un traitement antibiotique actif sur Legionella pneumophila. Il a permis une prise en charge adaptée et précoce des légionelloses et a contribué de ce fait à la diminution de la mortalité [10]. Cependant, il ne permet de diagnostiquer que Legionella pneumophila sérogroupe 1 (la plus souvent en cause), au risque de méconnaitre les autres légionelloses [9]. Les antibiotiques avec une pénétration intracellulaire constituent la base du traitement de la légionellose. Parmi ceux-ci, on retrouve les macrolides, les quinolones, les tétracyclines et la rifampicine. La stratégie thérapeutique est la suivante [9] : la forme non grave (patient ambulatoire, ou hospitalisé aux urgences ou en médecine) est traitée par monothérapie par macrolide, la forme grave (patient hospitalisé en unité de soins intensifs ou en réanimation, patient 
immunodéprimé) est traitée soit par monothérapie par fluoroquinolone soit par une association de deux antibiotiques entre macrolides, fluoroquinolones et rifampicine. La durée de traitement est de 8 à 14 jours pour les formes légères et de 14 à 21 jours pour les formes sévères [7-10]. Le pronostic de la légionellose dépend de l'hôte, de la précocité du diagnostic et de la rapidité d'instauration d'un traitement adapté ainsi que de la forme de la maladie [10].

En conclusion, la pneumonie à Legionella pneumophila est une maladie communautaire, rare, sévère et parfois mortelle. Les moyens diagnostiques rapides tels que la recherche de l'antigène urinaire soluble ont permis une prise en charge plus rapide et une amélioration de son diagnostic. Les cliniciens doivent savoir l'évoquer précocement, notamment en cas de pneumonie avec des signes extrapulmonaires comme la rhabdomyolyse et l'insuffisance rénale aiguë, afin de ne pas retarder l'administration des antibiotiques adaptés.

Liens d'intérêts : les auteurs déclarent ne pas avoir de liens d'intérêts.

\section{Références}

1. Santé publique France (2018) Distribution selon le mois de début des signes des cas de légionellose survenus en France (20162017). http://invs.santepubliquefrance.fr/Dossiers-thematiques/
Maladies-infectieuses/Maladies-a-declaration-obligatoire/Legionellose/Donnees-de-surveillance (Dernier accès le 23 juin 2018)

2. Benhamou D, Bru JP, Chidiac C, et al (2005) Légionellose : définition, diagnostic et traitement. Med Mal Inf 35:1-5

3. Farnham A, Alleyne L, Cimini D, Balter S (2014) Legionnaires' disease: incidence and risk factors, New York, USA, 2002-2011. Emerg Infect Dis 20:1795-802

4. Nicolini A, Ferraioli G, Senarega R (2013) Severe Legionella pneumophila pneumonia and non invasive ventilation: presentation of two cases and brief review of the literature. Pneumonol Alergol Pol 81:399-403

5. Sposato B, Mariotta S, Ricci A, et al (2003) Legionnaires' pneumonia with rhabdomyolysis and acute renal failure: a case report. Recenti Prog Med 94:391-4

6. Riou B, Richard C, Teboul JL, et al (1985). Atteinte musculaire au cours de la maladie des légionnaires : revue de la littérature à propos de 2 observations. Rev Med Int 6:105-10

7. Labidi J, Fdhila W, Battikh R, et al (2006) Pneumonie à légionelle compliquée d'une insuffisance rénale par rhabdomyolyse : à propos d'un cas. Med Mal Inf 36:476-8

8. Jamilloux Y, Jarraud S, Lina G, et al (2012) Legionella, légionellose. Med Sci 28:639-45

9. Comité des référentiels de la SPILF (2012) Le traitement antibiotique de la légionellose chez l'adulte. http://www.infectiologie. com/UserFiles/File/medias/_documents/consensus/2011-afssapsMAP-legionellose.pdf (Dernier accès le 23 juin 2018)

10. Société de pneumologie de langue française (SPLF); Société de pathologie infectieuse de langue française (SPILF) ; Centre national de référence sur les légionelles (2004) Légionellose : définition, diagnostic et traitement. http://www.infectiologie.com/ UserFiles/File/medias/_documents/legionelle-SPILF-aout2004.pdf (Dernier accès le 23 juin 2018) 